\title{
Outcomes following interventions to sustain body weight in esophageal cancer patients starting preoperative therapy: a retrospective cohort study
}

\author{
Jeffrey B. Velotta ${ }^{1}$, Jennifer R. Dusendang ${ }^{2}$, Hyunjee Kwak ${ }^{1}$, Michelle Huyser ${ }^{1}$, Ashish Patel ${ }^{1}$, \\ Simon K. Ashiku ${ }^{1}$, Lisa J. Herrinton ${ }^{2}$ \\ ${ }^{1}$ Department of Thoracic Surgery, Oakland Medical Center, Kaiser Permanente Northern California, Oakland, CA, USA; ${ }^{2}$ Division of Research, \\ Kaiser Permanente Northern California, Oakland, CA, USA \\ Contributions: (I) Conception and design: JB Velotta, LJ Herrinton; (II) Administrative support: JR Dusendang; (III) Provision of study materials or \\ patients: None; (IV) Collection and assembly of data: JR Dusendang, LJ Herrinton; (V) Data analysis and interpretation: All authors; (VI) Manuscript \\ writing: All authors; (VII) Final approval of manuscript: All authors. \\ Correspondence to: Lisa J. Herrinton. 2000 Broadway Ave., Oakland, CA 94612, USA. Email: lisa.herrinton@kp.org.
}

Background: To improve nutritional status and dysphagia, esophageal cancer patients starting neoadjuvant therapy in advance of curative-intent surgery may receive a jejunostomy tube (J-tube) or esophageal stent, or they may be managed without a feeding modality. We examined percent total weight loss (\% TWL), reinterventions, and progression to surgery in relation to these options.

Methods: The retrospective cohort study included stage II-III esophageal cancer patients diagnosed during 2010-2017 who received J-tube, stent, or nutritional counseling only, without a procedure, when starting chemotherapy or combined modality chemoradiation. Data were obtained from the electronic medical record and chart review. We compared median \% TWL between intervention groups and reinterventions using Chi-square and Kruskal-Wallis tests.

Results: Among the 366 patients, median \% TWL reached a nadir at 120 days, when it was $7 \%$ for patients with no procedure $(\mathrm{N}=307), 4 \%$ for $\mathrm{J}$-tube $(\mathrm{N}=39)$, and $16 \%$ for stent $(\mathrm{N}=20)(\mathrm{P}=0.01)$. Individual case analysis revealed $72-80 \%$ of the patients in the three groups started chemotherapy or chemoradiation as neoadjuvant curative-intent therapy ( $\mathrm{P}$ difference $=0.79$ ). In J-tube patients, the reasons for intervention was anticipation of weight loss in $49 \%$ and mitigation of actual weight loss in $15 \%$, whereas $95 \%$ of stent patients received the stent for dysphagia $(\mathrm{P}<0.001)$. A complication of the procedure was recorded in $85 \%$ of stent patients and $74 \%$ of $\mathrm{J}$-tube patients $(\mathrm{P}<0.001)$. Among those who received no procedure initially, 25\% received one later, compared with $15 \%$ of J-tube patients and $70 \%$ of stent patients who received a second procedure $(\mathrm{P}<0.001)$. Progression to surgery was observed in $65 \%$ of patients with no procedure, $51 \%$ of patients with J-tube, and $40 \%$ of stent patients, $\mathrm{P}=0.28$ ).

Conclusions: For stage II-III esophageal cancer patients starting chemotherapy, this study gives evidence that stents were associated with significant \% TWL and risk of reintervention. Although J-tube patients returned to baseline weight sooner than those with no procedure, they experienced complications from their J-tubes. For esophageal cancer patients undergoing curative-intent treatment and with acceptable levels of weight loss, no procedure at all may be superior to placing a J-tube in terms of complications, weight loss, and progression to curative-intent surgery.

Keywords: Esophageal cancer; chemotherapy; weight loss; community-based studies; care delivery

Submitted Nov 09, 2020. Accepted for publication Mar 03, 2021.

doi: $10.21037 /$ jtd-20-3220

View this article at: https://dx.doi.org/10.21037/jtd-20-3220 


\section{Introduction}

Esophageal cancer patients who are eligible for curativeintent surgery usually receive neoadjuvant chemotherapy or combined modality chemoradiation for tumor debulking and downstaging. Consequently, they may wait several months before surgery. Obstruction of the esophagus and gastroesophageal junction combined with the adverse effects of chemoradiation often result in weight loss (1), although knowledge of weight loss among those starting neoadjuvant therapy is inadequate (2-7). To improve nutritional status and to manage dysphagia, patients intended for surgical cure who start neoadjuvant therapy may receive a jejunostomy tube (J-tube) or esophageal stent, or may receive nutritional counseling with no procedural intervention.

The effectiveness and safety of $\mathrm{J}$-tube and stent for preventing weight loss has been summarized in a systematic review by Huddy and colleagues, although nutritional counseling alone was not evaluated (3). The authors concluded: "This review highlights the lack of an accepted standard of care or level 1 evidence in determining the optimal nutritional approach in patients with resectable loco-regional esophageal cancer undergoing neoadjuvant therapy". They further identified the need for information from observational cohort studies to determine the number of patients who may be potentially eligible and to collect evidence of current treatment decision-making. To fill this gap, we conducted a retrospective cohort study in a well-characterized, community-based population of Stage II-III esophageal cancer patients starting chemotherapy or combined modality chemoradiation for curative-intent who received a J-tube, esophageal stent, or no procedural intervention. We hypothesized that nutritional counseling alone may by the optimal nutrition approach with respect to percent total weight loss (\% TWL), complications and reinterventions, and progression to surgery.

We present the following article in accordance with the STROBE reporting checklist (available at https://dx.doi. org/10.21037/jtd-20-3220).

\section{Methods}

\section{Setting}

Kaiser Permanente Northern California is an integrated healthcare system serving 4.4 million patients, the great majority of whom receive capitated care. Esophageal cancer patients starting neoadjuvant chemotherapy who can tolerate at least a liquid diet usually receive nutritional counseling only with no procedure or surgical intervention. If they cannot tolerate both liquids and solids, then they are evaluated by the surgeon to receive either a stent or $\mathrm{J}$-tube. The decision is made by surgeon preference. The institution has not provided an algorithm or guideline. Most $\mathrm{J}$-tubes are performed laparoscopically, although this is also the surgeon's preference.

\section{Key elements of study design}

The exposure variable was nutritional intervention and the outcome was \% TWL. During a preliminary data analysis (Appendix 1), we noted that most nutritional interventions were performed at the start of chemotherapy or chemoradiation, or shortly thereafter, before the patient lost weight. We further noted that interventions performed later appeared to be in response to actual weight loss. To remove bias relating to the timing of the intervention, we required the intervention (nutritional counseling only, J-tube, stent) to occur before the start of chemotherapy or combined modality chemoradiation or within the following 10 days, and we defined the date of starting therapy as the index date. We allowed the intervention to occur within the 10 days after starting therapy to allow for interventions that were scheduled but not performed until after therapy began. Procedures performed 11 or more days after the date of starting therapy were defined as second procedures and were analyzed as outcomes. Thus, to remove bias related to the timing of the intervention, patients who had no procedure within the 10 days after their index date who had a later J-tube or stent were classified as having no procedure, with the later J-tube or stent classified as a re-intervention. Patients who received their first J-tube or stent more than 10 days after starting adjuvant therapy were reclassified as receiving no prophylactic intervention, with their later intervention noted as such, thus enabling an intention-to-treat analysis.

\section{Study population}

Adult Kaiser Permanente Northern California members with AJCC $8^{\text {th }}$ edition pathologic diagnosis of stage II-III esophageal cancer during 2010-17 who started chemotherapy or combined modality chemoradiation within 180 days of pathologic diagnosis were eligible for the study. We required at least one year of baseline enrollment before diagnosis to assure information on baseline factors. We excluded patients who had surgery before chemotherapy 
because these represented emergency procedures, and we excluded patients who received G-tube because these generally are not placed in patients intended for curativeintent surgery. Information about the cancer site, pathologic diagnosis, and stage was obtained from the health plan's cancer registry. Chemotherapy data were obtained from infusion data while radiation therapy was obtained from outpatient procedures.

\section{Data collection}

From the electronic medical record, information was obtained from the comprehensive electronic medical record for as long as 180 days after starting chemotherapy or combined modality chemoradiation (the index date). Information about $\mathrm{J}$-tubes was obtained from procedure codes recorded in inpatient and outpatient data. Information about stents required manual chart review of endoscopy and clinical notes containing the word "stent". The primary outcome was percent total weight loss (\%TWL). From vitals, we obtained every weight measurement from as long as 24 months before the date of pathologic diagnosis through 180 days after the index date. Initial body mass index $\left(\mathrm{kg} / \mathrm{m}^{2}\right)$ was calculated using the average of all height measurements and the most recent weight measurement recorded during the 2 years before the diagnosis. One patient with no procedural intervention and one with a J-tube had no weight measurement preceding their diagnosis, and for them, we used their first measurement after diagnosis. Weight data are prone to single digit recording errors, such as coding 150 pounds as 250 pounds. We therefore cleaned the weight data by removing measurements indicating that the patient had gained or lost more than 2 pounds per day. Reinterventions were obtained from procedure codes and chart review. Progression to surgery was obtained from procedure codes for esophagectomy or esophagogastrectomy, as was pneumonia (J12-J18) as a complication of procedure. Demographic characteristics were extracted from membership data. Comorbidities from clinical data recorded in the year before diagnosis, and tobacco usage from data recorded up to two years before diagnosis. Hospice transfers were identified from notes, and deaths from internal sources as well as state, National Death Index, and Social Security data.

Chart review was performed to confirm that the chemotherapy or combined modality chemoradiation was intended as neoadjuvant therapy, to capture complications from J-tube and stent, to assess whether the patient was intended for surgery, and reasons the patient did not progress to surgery. Because of the focus on device complications, and because patients without an intervention cannot have device complications, the chart review included all 39 J-tube patients, all 20 stent patients, but a random sample of 20 patients with no procedure. The charts were reviewed from the date of intervention to the end of the follow-up period, as defined in the follow paragraph. For the no-intervention group, the chart was reviewed starting 15 days before the start of chemotherapy, with 15 days being the median time from intervention to start of chemotherapy in the J-tube and stent patients.

\section{Statistical analysis}

Patients were classified into three groups (no procedure, J-tube, stent) as described above. Follow-up for outcomes began on the index date. Follow-up ended on the earliest of surgery, transfer to hospice, death, or 180 days, with no patient disenrolling from the health plan by 180 days. Outcomes included \% TWL, complications and reinterventions, and progression to surgery. We plotted median \% TWL from before the diagnosis date to the end of follow-up using every available weight measurement. We also computed median \% TWL at the index date (zero), 60,120 , and 180 days relative to their pre-diagnostic weight. The dataset used to evaluate \% TWL included one weight measurement for every patient for every day from the diagnosis date through the end of follow-up, by carrying forward each weight measurement to the next clinical assessment, at which time it was updated. We used the Kruskal-Wallis rank sum test to estimate $\mathrm{P}$ values for differences in median \% TWL across interventions. For reinterventions, and progression to surgery within 180 days, we computed $\mathrm{P}$ values using chi-square and Fisher's exact tests.

This study was deemed exempt by the Kaiser Permanente Northern California Institutional Review Board, IRB\# 00001045. The study was conducted in accordance with the Declaration of Helsinki (as revised in 2013). Individual consent for this retrospective analysis was waived.

\section{Results}

We identified 550 adults with stage II-III esophageal cancer diagnosed from 2010 to 2017. We excluded 21 (4\%) patients without pathological confirmation of cancer, 39 (7\%) without a full year of prior health-plan enrollment, 83 
(15\%) who did not receive chemotherapy or chemoradiation within 180 days after pathologic confirmation of cancer, and $6(1 \%)$ with surgery before chemotherapy, and 35 who received a G-tube, leaving 366 eligible patients. Among these, $307(84 \%)$ had nutritional counseling with no procedure before starting therapy (index date) or within the following 10 days. Another 39 (11\%) had a J-tube and $20(5 \%)$ had a stent. Chart review confirmed that 28 of 39 (72\%) J-tube patients, 16 of 20 stent patients ( $80 \%$ ), and 15 of $20(75 \%)$ no-intervention patients received preoperative and not definitive neoadjuvant chemotherapy $(\mathrm{P}=0.79)$. Of the $39 \mathrm{~J}$-tubes, 4 (10\%) were performed as open procedures and $35(90 \%)$ laparoscopically.

Characteristics of the 366 eligible patients are shown in Table 1. Over time, the frequency of using no procedure increased from 30 per year to about 45 per year $(\mathrm{P}<0.001)$, while use of J-tube declined from 6 per year to about 1 per year and use of stent increased from about 1 per year to 6 per year. The choice of intervention differed in relation to smoking status $(\mathrm{P}=0.02)$. Median \% TWL between the diagnosis and index dates was $0.7 \%$ for patients with no intervention, $2.6 \%$ for J-tube patients, and $4.1 \%$ for stent patients $(\mathrm{P}<0.01)$.

The median time from the intervention to the start of therapy was 21 days for stent and 11 days for J-tube. The average number of weight measurements per patient was similar across groups (4.5 to 6.0 between diagnosis and index; 13.2 to 16.6 from index to the end of followup). After the index date, the median \% TWL at the nadir (120 days) was $7.0 \%$ for no procedure, $4.3 \%$ for J-tube, and $16.3 \%$ for stent $(\mathrm{P}=0.01)$ (Table 2). Patients with no procedure or with $\mathrm{J}$-tube more often progressed to surgery or to the end of the study while those with stent more often were transferred to hospice or died (compared with no procedure: J-tube, $\mathrm{P}=0.25$; stent, $\mathrm{P}<0.01$ ).

The median time from pathologic diagnosis to the index date was similar (35-39 days) across the three groups. The median time from the intervention to the start of therapy was 21 days for stent and 11 days for J-tube. The average number of weight measurements per patient was similar across groups ( 4.5 to 6.0 between diagnosis and index; 13.2 to 16.6 from index to the end of follow-up). Figure 1 plots the time course of median \% TWL in relation to the intervention. Zero on the $\mathrm{X}$-axis represents the date of starting therapy. Stents were placed a median 21 days before the index date and J-tubes placed about 10 days later. Weight loss reached a nadir at about 120 days after starting therapy, with stent patients having the greatest \% TWL.
The plot suggests recovery of body weight beginning at about 120 days, with J-tube patients have more rapid and complete recovery than patients without an intervention.

Pneumonia was diagnosed in 7/39 (18\%) J-tube patients, $7 / 20(35 \%)$ stent patients, and 53/307 no-intervention patients $(17 \%)(\mathrm{P}=0.18)$. The results of the chart review are provided in Table 3. Compared with no procedure or J-tube, stent patients had somewhat lower tumors, although the difference was not statistically significant $(\mathrm{P}=0.49)$. The groups were similar in that about three-quarters of the patients in each group received therapy as adjuvant therapy $(\mathrm{P}=0.79)$. One stent patient had a fistula. No other patient was documented with a fistula before intervention. Most had dysphagia, although $25 \%$ of those with no intervention did not have dysphagia. The reasons for intervention differed between $\mathrm{J}$-tube and stent in that $\mathrm{J}$-tubes were placed in $49 \%$ of patients in anticipation of weight loss and in $15 \%$ to mitigate actual weight loss, whereas most stent patients received the stent for dysphagia $(\mathrm{P}<0.001)$. The table also tabulates complications following the index date, with $85 \%$ of stent patients, $74 \%$ of J-tube patients, and $5 \%$ of patients with no procedure having a complication $(\mathrm{P}<0.001)$. Among those who initially did not undergo a procedure, $25 \%$ underwent a procedure $\geq 11$ days after the index date compared with $15 \%$ of J-tube patients and $70 \%$ of stent patients $(\mathrm{P}<0.001)$.

\section{Discussion}

We examined a community-based cohort of 366 stage II-III esophageal cancer patients diagnosed during 2010-2017 and starting chemotherapy or chemoradiation, of whom $11 \%$ received J-tube, $5 \%$ stent, and $84 \%$ no procedure at the time of starting their therapy. The key study finding was the similarity in \% TWL between patients who received no procedure and those who received a J-tube. Patients with a J-tube recovered slightly more weight between 120 and 180 days, but the difference in \%TWL from baseline of $5.3 \%$ with no procedure vs. $3.6 \%$ with J-tube was minimal. Key strengths of our study were the restriction to stage II-III esophageal cancer patients and use of an intention-to-treat analysis. In the present study, the J-tube patients were quite similar in baseline characteristics to those with no procedure, with $72-80 \%$ receiving neoadjuvant therapy and thus planned for curative-intent surgery and patients having minimal \% TWL before starting preoperative therapy. Although J-tube patients recovered their weight more rapidly than patients without 
Table 1 Baseline characteristics of adults with esophageal cancer in relation to nutritional procedure received up to 10 days after the index date (start of neoadjuvant therapy). Kaiser Permanente Northern California, 2010-2017

\begin{tabular}{|c|c|c|c|c|c|c|c|}
\hline \multirow[t]{2}{*}{ Baseline characteristic } & \multicolumn{2}{|c|}{$\begin{array}{c}\text { Nutritional counseling with no } \\
\text { procedure, } \mathrm{N}=307\end{array}$} & \multicolumn{2}{|c|}{ J-tube, $N=39$} & \multicolumn{2}{|c|}{ Stent, $N=20$} & \multirow[t]{2}{*}{$P$ value } \\
\hline & $\mathrm{N}$ & $\%$ & $\mathrm{~N}$ & $\%$ & $\mathrm{~N}$ & $\%$ & \\
\hline \multicolumn{8}{|l|}{ Year of cancer diagnosis } \\
\hline 2010-2012 & 91 & 30 & 19 & 49 & 2 & 10 & $<0.001$ \\
\hline 2013-2015 & 126 & 41 & 17 & 44 & 6 & 30 & \\
\hline 2016-2017 & 90 & 29 & 3 & 8 & 12 & 60 & \\
\hline \multicolumn{8}{|l|}{ Histologic type } \\
\hline Adenocarcinoma & 189 & 62 & 26 & 67 & 13 & 65 & $0.77^{\star \star}$ \\
\hline Squamous cell & 96 & 31 & 12 & 31 & 7 & 35 & \\
\hline Other & 22 & 7 & 1 & 3 & 0 & 0 & \\
\hline \multicolumn{8}{|l|}{ Stage at diagnosis } \\
\hline II & 96 & 31 & 15 & 38 & 9 & 45 & 0.33 \\
\hline III & 211 & 69 & 24 & 62 & 11 & 55 & \\
\hline \multicolumn{8}{|l|}{ Age at diagnosis, years } \\
\hline $18-59$ & 58 & 19 & 7 & 18 & 5 & 25 & $0.21^{\star \star}$ \\
\hline $60-69$ & 108 & 35 & 20 & 51 & 7 & 35 & \\
\hline $70-79$ & 108 & 35 & 12 & 31 & 7 & 35 & \\
\hline $80-89$ & 33 & 11 & 0 & 0 & 1 & 5 & \\
\hline \multicolumn{8}{|l|}{ Gender } \\
\hline Male & 241 & 79 & 31 & 79 & 13 & 65 & 0.36 \\
\hline Female & 66 & 22 & 8 & 21 & 7 & 35 & \\
\hline \multicolumn{8}{|l|}{ Race/ethnicity } \\
\hline Asian & 33 & 11 & 2 & 5 & 0 & 0 & $0.61^{\star \star}$ \\
\hline Black & 13 & 4 & 1 & 3 & 1 & 5 & \\
\hline Hispanic, any race & 28 & 9 & 2 & 5 & 2 & 10 & \\
\hline White & 210 & 68 & 32 & 82 & 17 & 85 & \\
\hline Other & 23 & 7 & 2 & 5 & 0 & 0 & \\
\hline \multicolumn{8}{|l|}{ Body mass index ${ }^{*}, \mathrm{~kg} / \mathrm{m}^{2}$} \\
\hline Underweight (<18.5) & 7 & 2 & 0 & 0 & 2 & 10 & 0.20 \\
\hline Healthy weight (18.5-24.9) & 100 & 33 & 10 & 26 & 5 & 25 & \\
\hline Overweight (25-29.9) & 97 & 32 & 15 & 38 & 4 & 20 & \\
\hline Obese $(30+)$ & 103 & 34 & 14 & 36 & 9 & 45 & \\
\hline \multicolumn{8}{|l|}{ Smoking } \\
\hline Ever & 210 & 68 & 22 & 56 & 18 & 90 & $0.02^{\star *}$ \\
\hline Never & 91 & 30 & 13 & 33 & 2 & 10 & \\
\hline Unknown & 6 & 2 & 4 & 10 & 0 & 0 & \\
\hline \multicolumn{8}{|l|}{ Charlson comorbidity } \\
\hline 0 & 106 & 35 & 14 & 26 & 4 & 20 & $0.71^{\star *}$ \\
\hline $1-2$ & 173 & 56 & 21 & 60 & 14 & 70 & \\
\hline$\geq 3$ & 28 & 9 & 4 & 14 & 2 & 10 & \\
\hline Median \%TWL from diagnosis to index & & $0.7 \%$ & & $2.6 \%$ & & $4.1 \%$ & $<0.01$ \\
\hline
\end{tabular}

${ }^{*}$, most recent measurement in the 2 years preceding diagnosis; ${ }^{* \star}$, P values were computed from chi-square test, Fisher's exact test for small cell counts, and Kruskal-Wallis test for medians. 
Table $2 \%$ TWL after the start of chemotherapy or chemoradiation in esophageal cancer patients in relation to nutritional procedure (intentionto-treat analysis). Kaiser Permanente Northern California, 2010-2017

\begin{tabular}{|c|c|c|c|c|}
\hline Median $\%$ total weight loss (\%TWL) & Nutritional counseling with no procedure, $\mathrm{N}=307$ & J-tube, $N=39$ & Stent, $\mathrm{N}=20$ & $P$ value $e^{\star \star}$ \\
\hline From index to 120 days later & $7.0 \%$ & $4.3 \%$ & $16.3 \%$ & 0.01 \\
\hline From index to 180 days later & $5.3 \%$ & $3.6 \%$ & $13.2 \%$ & 0.03 \\
\hline
\end{tabular}

*, follow-up for outcomes began on the index date (the date of starting neoadjuvant therapy) and ended on the earliest of surgery, transfer to hospice, death, or 180 days, with no patient disenrolling from the health plan by 180 days. The median time from the intervention to the start of therapy was 21 days for stent and 11 days for $\mathrm{J}$-tube. The average number of weight measurements per patient was similar across groups ( 4.5 to 6.0 between diagnosis and index; 13.2 to 16.6 from index to the end of follow-up). The timing of interventions performed 1-10 days after the index date were coded using negative numbers. No patient disenrolled by 180 days. ${ }^{\star *}$, estimated from the KruskalWallis test.

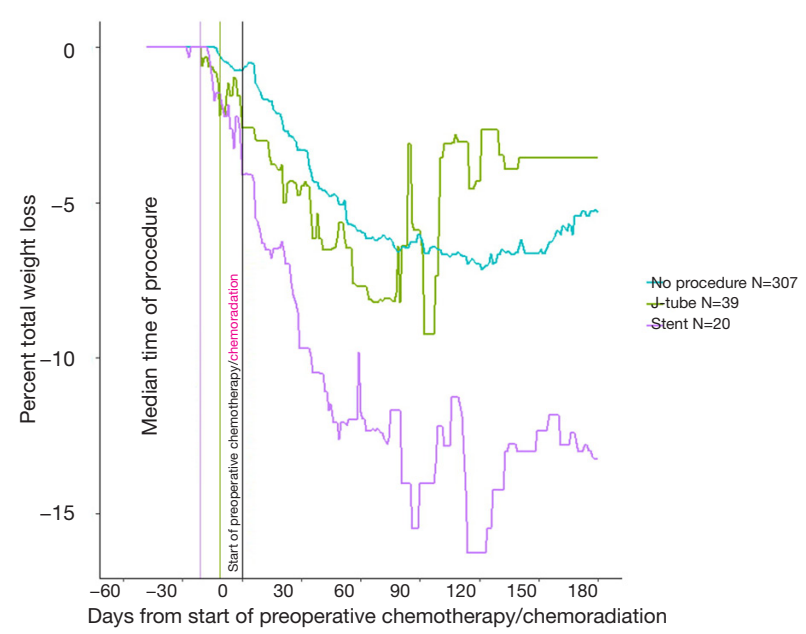

Figure 1 Within-person percent total weight loss following the diagnosis of esophageal cancer in relation to nutritional procedure received up to 10 days after the start of neoadjuvant therapy. Kaiser Permanente Northern California, 2010-2017. Zero on the X-axis represents the date of starting neoadjuvant therapy. The colored vertical lines crossing the $\mathrm{X}$-axis show the median timing of intervention, with stents placed a median 21 days before the index date and J-tubes placed about 10 days later. Patients were grouped based on nutritional procedure they received within 10 days after their index date. The median time to intervention is shown by the three colored vertical lines. Patients were followed from the date of pathologic confirmation of esophageal cancer to the earlier of surgery, transfer to hospice, death, or 180 days after the index date, with no patient having disenrolled by 180 days. The dataset used to construct this plot included a weight measurement for every day of follow-up by carrying forward the most recent value to the next measurement. an intervention, the difference was modest. J-tube patients had significant complications from their procedure. In this intention-to-treat analysis, only $25 \%$ of the no-procedure group went on to have an intervention.,. Some patients had second interventions, most often because of excess weight loss or complications, that mediated their subsequent likelihood of an adverse outcome, and this was most striking for stent patients who later had a J-tube.

Regarding no intervention versus J-tube, Tsujimoto and colleagues studied $9 \mathrm{~J}$-tube patients and 56 controls with esophageal cancer who received neoadjuvant chemotherapy, noted that J-tube patients gained an average of $0.4 \mathrm{~kg}$ while controls lost an average of $1.9 \mathrm{~kg}$ after chemotherapy (6). Jenkins and colleagues (7) compared patients (2007-14) who underwent neoadjuvant chemoradiation and received, prior to surgery, no procedure $(\mathrm{N}=49)$ versus any intervention (32 J-tube; 14 gastrostomy tube; 1 stent; 1 nasoenteric tube; 2 combination). The \% TWL within 6 months of esophagectomy averaged $15.4 \%$ in those with no procedure and $11.2 \%$ in those with an intervention $(\mathrm{P}=0.1)$. Jenkinson and colleagues (4) described 35 patients who received a J-tube and preoperative chemotherapy, of whom $32 \%$ required immediate feeding and 14\% later feeding before surgery, with the J-tube patients gaining an average of $1.1 \mathrm{~kg}$ in advance of surgery. A systematic review by Deftereos and colleagues (8) summarized 443 esophageal cancer patients, noting that dietary counseling and enteral feeding in neoadjuvant therapy was effective for weight maintenance and surgical complications, although the quality of evidence was low. A 2010 review article recommended dietetic surveillance and oral supplementation 
Table 3 Chart review of 79 adults with esophageal cancer in relation to nutritional procedure received up to 10 days after the start of neoadjuvant therapy. Kaiser Permanente Northern California, 2010-2017

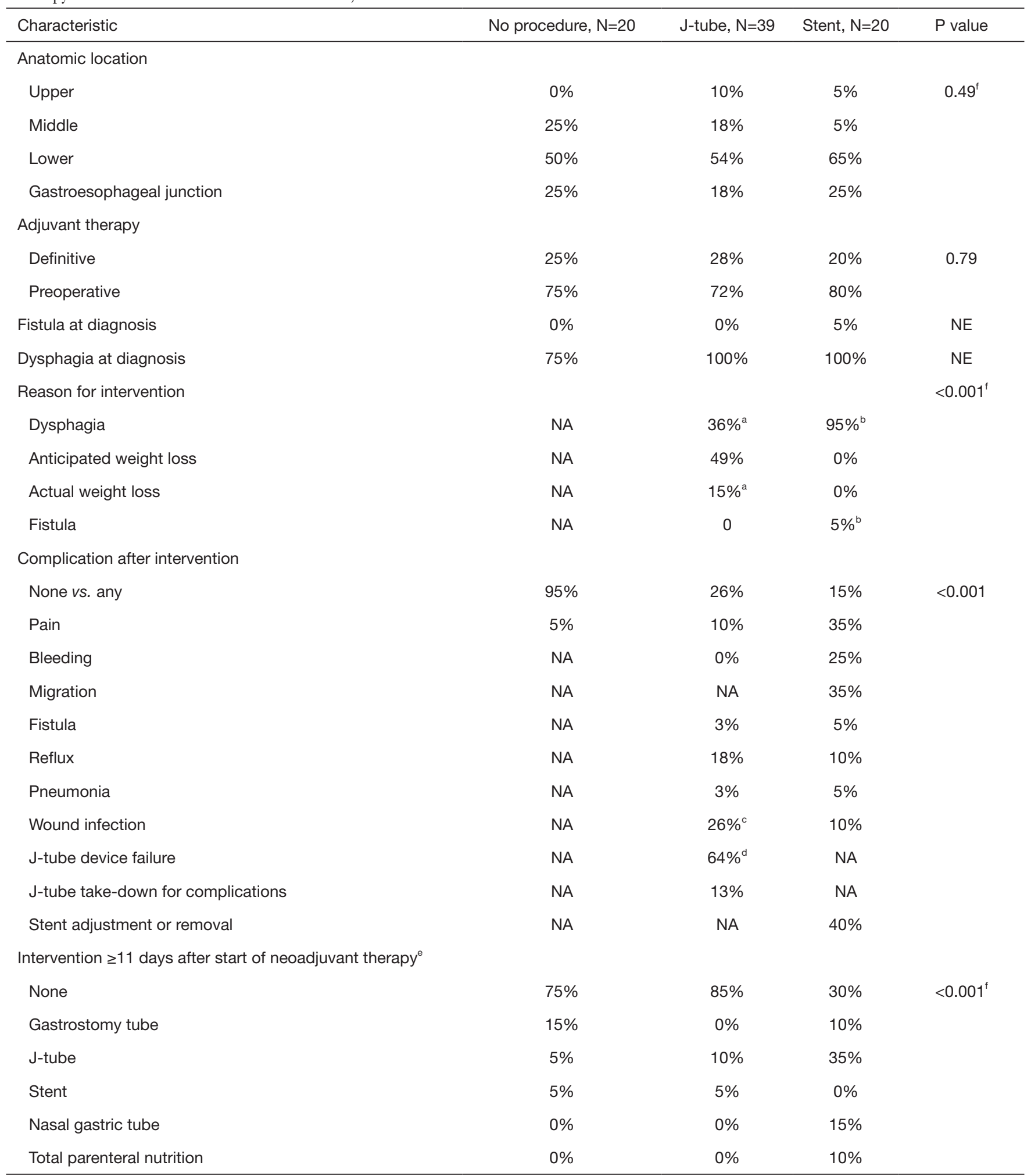

Table 3 (continued) 
Table 3 (continued)

\begin{tabular}{|c|c|c|c|c|}
\hline Characteristic & No procedure, $\mathrm{N}=20$ & J-tube, $N=39$ & Stent, $N=20$ & $P$ value \\
\hline Yes & $65 \%$ & $51 \%$ & $40 \%$ & 0.28 \\
\hline No, death & $0 \%$ & $5 \%$ & $10 \%$ & \\
\hline No, disease not cleared & $5 \%$ & $3 \%$ & $0 \%$ & \\
\hline No, patient not fit for surgery & $5 \%$ & $13 \%$ & $25 \%$ & \\
\hline No, bowel perforation from J-tube & $0 \%$ & $3 \%$ & $0 \%$ & \\
\hline No, patient preference & $0 \%$ & $0 \%$ & $5 \%$ & \\
\hline No, surveillance & $15 \%$ & $10 \%$ & $0 \%$ & \\
\hline
\end{tabular}

${ }^{a}$, one patient had both dysphagia and actual weight loss and is counted twice; ${ }^{b}$, one patient had dysphagia and fistula and is counted twice; ${ }^{c}$, included 9 wound infections and one urinary tract infection; ${ }^{d}$, device failures included clogging, falling out, and plug and cap problems; ${ }^{e}$, for patients classified with no procedure at entry into follow-up, this was their first intervention. For patients classified with j-tube or stent at entry into follow-up, this was their second intervention; ${ }^{\dagger}$, Fisher's exact test was used in place of chi square because of small cell counts. NE, not estimated; NA, not applicable.

for non-dysphagic esophageal cancer patients receiving neoadjuvant treatment, and enteral feeding for dysphagic patients (9). In a single institution study of 99 neoadjuvant chemotherapy patients, half receive enteral access prior to chemoradiation and half at the time of surgery. Although the patients were not randomized, they had similar clinicopathological characteristics.

Stent patients in this study lost more weight than the other groups, had more complications, and less frequently went on to curative-intent surgery. This result is similar to the systematic review of Ahmed and colleagues who found that although esophageal stents resulted in significant improvements in dysphagia scores, they did not improve overall body weight or albumin levels (2). Similar to this study, they found that relatively few stent patients (33\%) progressed to curative esophagectomy. In an observational study of 38 patients who received a stent and who were propensity-score matched to 152 patients who did not receive a stent, the stent group had worse oncologic outcomes including positive margins on resection (39\% vs. $15 \%, \mathrm{P}=0.04)$, shorter median time to recurrence (6.5 vs. 9 months, $\mathrm{P}=0.04)$, and worse 3 -year survival ( $25 \%$ vs. $44 \%$, $\mathrm{P}=0.02)(10)$. Esophageal stents have been associated with early life-threatening morbidity and higher mortality rates during concurrent neoadjuvant chemoradiation compared to no stent placement (11). In addition, radiation planning is affected by placement of self-expanding metallic stents (12), which cause radiation dose perturbations ranging from 0 to $30 \%$. Patients who have pain associated with stent placement and concurrent chemoradiation are difficult to manage as outpatients because their severe odynophagia precludes them from obtaining adequate oral nutrition even when the esophageal lumen remains patent. It may be that esophageal stenting is most useful for palliation of stage IV cancer, to provide relatively non-invasive oral comfort and treat dysphagia $(13,14)$. However, in patients with inoperable gastroesophageal junction cancers, Sihvo and colleagues reported that laser therapy had a more beneficial effect on dysphagia, with lower morbidity and mortality and without increased hospital costs related to stent complications (15).

A systematic review by Huddy and colleagues summarized observational studies for J-tube and stent, but did not assess nutritional counseling alone as an intervention (3). The authors found the evidence to be insufficient to establish a standard of care and identified the need for observational cohort studies to assess current treatment decision-making and the number of patients potentially eligible for randomized trials. National Comprehensive Cancer Network guidelines recommend against stent for patients with dysphagia who are candidates for curative surgery because of their adverse event profile (16). Our 
study affirms that stents are prone to complications and provides new details about weight outcomes following stent placement.

Given the results of past observational studies, and absent randomized trials, it has become common in our health care system not to place any intervention prior to neoadjuvant therapy of esophageal cancer. Indeed $76 \%$ of the study cohort received no procedure, with $25 \%$ of these needing a later intervention during their neoadjuvant course. This analysis of patients with no procedure suggests that no intervention is a reasonable initial nutritional strategy for curative-intent patients with the expectation that a minority of patients may need a J-tube at a later time. We recommend waiting rather than placing $\mathrm{J}$-tubes routinely as is common in some settings. In our system, placement of esophageal stents is now restricted to stage IV patients with severe dysphagia or esophageal fistula complications.

Despite the large underlying population, the sample size was relatively small, which limited the ability to perform detailed analyses. In addition, the study was not randomized. In contrast to randomized controlled trials, observational studies of treatment effectiveness and safety are considered lower quality evidence because of likely differences across study groups in the extent of disease and reason for intervention, i.e., selection bias. However, randomized controlled trials can be highly resource-intensive, and observational studies often are essential for designing randomized trials, because they can clarify characteristics of the eligible population and the degree of standardization of treatment, while also framing the expected magnitude of impact. Furthermore, when a randomized controlled trial is infeasible, well-executed observational studies can be used to develop guidelines after taking into consideration the likely magnitude of biases.

Regarding specific selection factors, the stent patients included in this study may well have differed from J-tube and no-procedure patients in disease severity and health status. However, patient age, stage, comorbidity, and reason for chemotherapy (definitive, preoperative) were similar across the three groups, if not slightly more favorable among the stent group. In addition, like patients in the other two groups, most of the stent patients received chemotherapy and chemoradiation prior to eventual curative-intent surgery. On the other hand, information on the severity of dysphagia and the patient's experience of the symptom were not available for the study and may have been worse for the stent patients. Even so, dysphagia would not explain the magnitude of weight loss, device complication, or failure to progress to surgery in the stent patients. A J-tube may have been more appropriate for these patients.

Despite limitations, this study provides evidence that most esophageal cancer patients starting curative-intent neoadjuvant therapy maintain adequate weight without any feeding device or stent. We have shown that few patients fail this regimen, and that \% TWL is similar between the nutritional counseling only and J-tube groups. Resisting J-tube placement significantly reduces complications such as clogging, kinking, and wound infections. A striking percentage of patients receiving stents had worsened $\%$ TWL, more procedural complications, and failed to progress to curative-intent surgery, either because of their esophageal cancer or because of their stent. We remain concerned that placement of preoperative esophageal stents for curative-intent surgical patients pose negative consequences, consistent with the National Comprehensive Cancer Network guidelines. These findings are generalizable and for esophageal cancer patients undergoing curative-intent treatment and with acceptable levels of weight loss, no procedure at all may be superior to placing a $\mathrm{J}$-tube in terms of complications, \%TWL, and progression to curative-intent surgery.

\section{Acknowledgments}

We wish to thank Dr. Stacey Alexeeff for biostatistical review.

Funding: This project was supported by The Permanente Medical Group Delivery Science and Applied Research initiative.

\section{Footnote}

Reporting Checklist: The authors have completed the STROBE reporting checklist. Available at http://dx.doi. org/10.21037/jtd-20-3220

Data Sharing Statement: Available at https://dx.doi. org/10.21037/jtd-20-3220

Peer Review File: Available at https://dx.doi.org/10.21037/ jtd-20-3220

Conflicts of Interest: All authors have completed the ICMJE uniform disclosure form (available at https://dx.doi. org/10.21037/jtd-20-3220). The authors are partners and 
staff of The Permanente Medical Group and report no other conflicts of interest.

Ethical Statement: The authors are accountable for all aspects of the work in ensuring that questions related to the accuracy or integrity of any part of the work are appropriately investigated and resolved. This study was deemed exempt by the Kaiser Permanente Northern California Institutional Review Board, IRB\# 00001045. The study was conducted in accordance with the Declaration of Helsinki (as revised in 2013). Individual consent for this retrospective analysis was waived.

Open Access Statement: This is an Open Access article distributed in accordance with the Creative Commons Attribution-NonCommercial-NoDerivs 4.0 International License (CC BY-NC-ND 4.0), which permits the noncommercial replication and distribution of the article with the strict proviso that no changes or edits are made and the original work is properly cited (including links to both the formal publication through the relevant DOI and the license). See: https://creativecommons.org/licenses/by-nc-nd/4.0/.

\section{References}

1. Reim D, Friess H. Feeding Challenges in Patients with Esophageal and Gastroesophageal Cancers. Gastrointest Tumors 2016;2:166-77.

2. Ahmed O, Bolger JC, O'Neill B, et al. Use of esophageal stents to relieve dysphagia during neoadjuvant therapy prior to esophageal resection: a systematic review. Dis Esophagus 2020;33:doz090.

3. Huddy JR, Huddy FMS, Markar SR, et al. Nutritional optimization during neoadjuvant therapy prior to surgical resection of esophageal cancer-a narrative review. Dis Esophagus 2018;31:1-11.

4. Jenkinson AD, Lim J, Agrawal N, et al. Laparoscopic feeding jejunostomy in esophagogastric cancer. Surg Endosc 2007;21:299-302.

5. Grondona P, Andreani SM, Barr N, et al. Laparoscopic feeding jejunostomy technique as part of staging laparoscopy. Surg Laparosc Endosc Percutan Tech 2005;15:263-6.

6. Tsujimoto H, Hiraki S, Takahata R, et al. Laparoscopic jejunostomy for obstructing upper gastrointestinal malignancies. Mol Clin Oncol 2015;3:1307-10.

7. Jenkins TK, Lopez AN, Sarosi GA, et al. Preoperative enteral access is not necessary prior to multimodality treatment of esophageal cancer. Surgery 2018;163:770-6.

8. Deftereos I, Kiss N, Isenring E, et al. A systematic review of the effect of preoperative nutrition support on nutritional status and treatment outcomes in upper gastrointestinal cancer resection. Eur J Surg Oncol 2020;46:1423-34.

9. Bozzetti F. Nutritional support in patients with oesophageal cancer. Support Care Cancer 2010;18 Suppl 2:S41-50.

10. Mariette C, Gronnier C, Duhamel A, et al. Self-expanding covered metallic stent as a bridge to surgery in esophageal cancer: impact on oncologic outcomes. J Am Coll Surg 2015;220:287-96.

11. Lu YF, Chung CS, Liu CY, et al. Esophageal Metal Stents with Concurrent Chemoradiation Therapy for Locally Advanced Esophageal Cancer: Safe or Not? Oncologist 2018;23:1426-35.

12. Tryggestad EJ, Liu W, Pepin MD, et al. Managing treatment-related uncertainties in proton beam radiotherapy for gastrointestinal cancers. J Gastrointest Oncol 2020;11:212-24.

13. Gray RT, O'Donnell ME, Scott RD, et al. Self-expanding metal stent insertion for inoperable esophageal carcinoma in Belfast: an audit of outcomes and literature review. Dis Esophagus 2011;24:569-74.

14. Dai Y, Li C, Xie Y, et al. Interventions for dysphagia in oesophageal cancer. Cochrane Database Syst Rev 2014;(10):CD005048.

15. Sihvo EI, Pentikäinen T, Luostarinen ME, et al. Inoperable adenocarcinoma of the oesophagogastric junction: a comparative clinical study of laser coagulation versus self-expanding metallic stents with special reference to cost analysis. Eur J Surg Oncol 2002;28:711-5.

16. National Comprehensive Cancer Network. NCCN Clinical Practice Guidelines in Oncology: Esophageal and esophagogastric junction cancers. Available online: https:// www.nccn.org/professionals/physician_gls/pdf/esophageal. pdf. Accessed May 4, 2020.

Cite this article as: Velotta JB, Dusendang JR, Kwak H, Huyser M, Patel A, Ashiku SK, Herrinton LJ. Outcomes following interventions to sustain body weight in esophageal cancer patients starting preoperative therapy: a retrospective cohort study. J Thorac Dis 2021;13(9):5477-5486. doi: 10.21037/ jtd-20-3220 


\section{Supplementary}

\section{Appendix 1}

Before defining the index date, we obtained random samples of 20 patients with each intervention and plotted their individual weight loss trajectories using \% total weight loss (TWL). The start date of neoadjuvant therapy is centered at zero, while the black vertical line is the date of intervention. To the right of zero, the blue line represents the date of surgery, light orange is transfer to hospice, dark orange is death, and green is the end of the study at 180 days. We observed that for some patients, late intervention followed rapid weight loss. After creating these plots, we decided to classify interventions based on events that occurred within 10 days of the start of neoadjuvant therapy to avoid confounding by late weight loss.
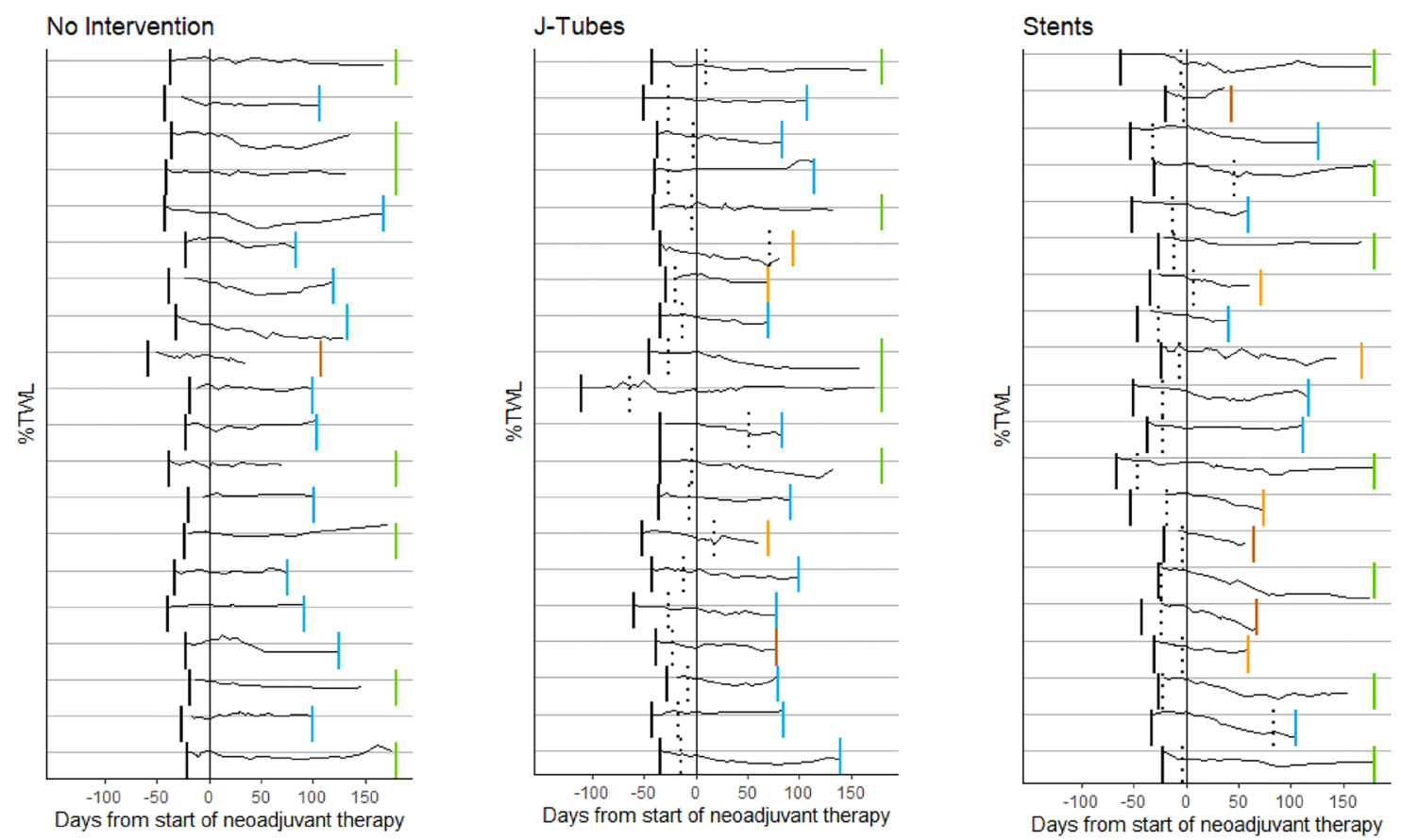